Research Article

\title{
Correlation between TCM Syndromes and Type 2 Diabetic Comorbidities Based on Fully Connected Neural Network Prediction Model
}

\author{
Yifei Wang, ${ }^{1}$ Runshun Zhang, ${ }^{2}$ Min Pi, ${ }^{3}$ Julia Xu, ${ }^{4}$ Moyan Qiu, ${ }^{1}$ and Tiancai Wen $\mathbb{D}^{5,6}$ \\ ${ }^{1}$ Wangjing Hospital, China Academy of Chinese Medical Sciences, Beijing, China \\ ${ }^{2}$ Guang'anmen Hospital, China Academy of Chinese Medical Sciences, Beijing, China \\ ${ }^{3}$ Shenzhen Traditional Chinese Medicine Hospital, Shenzhen, Guangdong, China \\ ${ }^{4}$ The University of Melbourne, Melbourne, Australia \\ ${ }^{5}$ Data Center of Traditional Chinese Medicine, China Academy of Chinese Medical Sciences, Beijing, China \\ ${ }^{6}$ Institute of Basic Research in Clinical Medicine, China Academy of Chinese Medical Sciences, Beijing, China \\ Correspondence should be addressed to Tiancai Wen; wentiancai@ndctcm.cn
}

Received 16 August 2021; Accepted 8 November 2021; Published 20 November 2021

Academic Editor: Mohammad Hashem Hashempur

Copyright (c) 2021 Yifei Wang et al. This is an open access article distributed under the Creative Commons Attribution License, which permits unrestricted use, distribution, and reproduction in any medium, provided the original work is properly cited.

Objective. To predict the major comorbidities of type 2 diabetes based on the distribution characteristics of syndromes, and to explore the relationship between TCM syndromes and comorbidities of type 2 diabetes. Methods. Based on the electronic medical record data of 3413 outpatient visits from 995 type 2 diabetes patients with comorbidities, descriptive statistical methods were used to analyze the basic characteristics of the population, the distribution characteristics of comorbidities, and TCM syndromes. A neural network model for the prediction of type 2 diabetic comorbidities based on TCM syndromes was constructed. Results. Patients with TCM syndrome of blood amassment in the lower jiao were diagnosed with renal insufficiency with $95 \%$ test sensitivity. The patients with spleen deficiency combined with ascending counterflow of stomach qi and cold-damp patterns were diagnosed with gastrointestinal lesions with $92 \%$ sensitivity. The patients with TCM syndrome group of spleen heat and exuberance of heart fire were diagnosed as type 2 diabetes complicated with hypertension with a sensitivity of $91 \%$. In addition, the prediction accuracy of combined neuropathy, heart disease, liver disease, and lipid metabolism disorder reached 70 90\% in TCM syndrome groups. Conclusion. The fully connected neural network model study showed that syndrome characteristics are highly correlated with type 2 diabetes comorbidities. Syndrome location is commonly in the heart, spleen, stomach, lower jiao, meridians, etc., while syndrome pattern manifests in states of deficiency, heat, phlegm, and blood stasis. The different combinations of disease location and disease pattern reflect the syndrome characteristics of different comorbidities forming the characteristic syndrome group of each comorbidity. Major comorbidities could be predicted with a high degree of accuracy through TCM syndromes. Findings from this study may have further implementations to assist with the diagnosis, treatment, and prevention of diabetic comorbidities at an early stage.

\section{Introduction}

Type 2 diabetes mellitus (T2DM) is a chronic and progressive disease with multiple etiologies over a long course. It seriously affects the quality of life of patients and increases the risk of early death [1]. IDF estimated that approximately 4.2 million adults will die as a result of diabetes and its complications in 2019. Globally, 11.3\% of deaths are due to diabetes. Almost half of these deaths are in people under
60 years of age [2]. Studies have found that more than $40 \%$ of diabetics suffer from comorbidities [3]. Comorbidity is an important factor affecting the prognosis of diabetics, and some comorbidities are also major causes of disability and death.

Type 2 diabetes develops mainly due to insulin resistance and relative insulin insufficiency and usually manifests as a glycolipid metabolism disorder $[4,5]$. As diabetes progresses over time, various comorbidities may develop. In recent 
years, many studies have shown that complementary and alternative medicine therapies including traditional Chinese medicine (TCM) have good efficacy in improving insulin resistance and regulating glycolipid metabolism with a high degree of safety [5-8]. At the same time, the application of relevant complementary alternative medicine therapies is of great significance in improving the quality of life of diabetic patients and preventing the occurrence and development of diabetes-related comorbidities. However, long-term hyperglycemia in diabetics affects multiple organs over time. Therefore, in the treatment of diabetes with complementary and alternative medicine, we must first accurately diagnose diabetes mellitus according to a theoretical system.

TCM is a common and important model of complementary and alternative medicine. It is the crystallization of thousands of years of medical experience and wisdom of the Chinese nation [9]. TCM has unique insights into the diagnosis and treatment of diabetes and its comorbidities and has been shown to effectively control the progression of diabetes, as well as diagnose, prevent, and treat diabetes mellitus at an early stage. TCM emphasizes the concepts of "holism" and "syndrome differentiation and treatment". "Syndrome differentiation" is the essence of TCM diagnosis and treatment of disease. TCM views that disease symptoms reflect the nature of a particular internal and external environment at a certain stage of the disease or the individual patient at the time. It manifests in corresponding patterns observed in the tongue, pulse, shape, color, and complexion. Patterns can also reveal aspects of pathogenesis such as etiology, disease location, disease nature, and disease status to various degrees, providing a basis for syndrome differentiation and treatment [10]. Different diseases contain different progression patterns of syndromes [11]. Syndromes usually reflect the current state of the disease. Being able to clarify the relationship between syndromes and various comorbidities will assist with the diagnosis of type 2 diabetes comorbidities based on syndrome characteristics, which is important for early treatment and prevention. Based on real clinical medical records, this study analyzed the relationship between TCM syndromes and comorbidities. A prediction model of "TCM syndrome-comorbidity" based on a neural network was also constructed to provide a reference for the diagnosis of diabetes comorbidity.

\section{Data and Methods}

2.1. Data Sources. The data for this study was obtained from the electronic medical records of T2DM patients, which were collected in the database of the Data Center of Traditional Chinese Medicine, China Academy of Chinese Medical Sciences. Data screening rules were as follows: (1) the primary diagnosis being type 2 diabetes, (2) at least one secondary diagnosis, (3) age $\geq 18$ years, and (4) complete information about TCM and Western medicine diagnosis and TCM syndrome diagnosis. To protect privacy, all personal information such as patient names and phone numbers was desensitized prior to the study, and medical record numbers were used to identify each patient. Data regarding patient ID, gender, age, frequency of visit, course of the disease, disease diagnosis, and syndrome diagnosis was extracted. This study was approved by the Ethics Committee of the Institute of Basic Research in Clinical Medicine, China Academy of Chinese Medical Sciences (approval number: 2016 No.11-01).

2.2. Data Standardization and Classification. In cases where disease names were not standardized (terms have different semantic expressions), medical professionals matched the original data with the standard disease names in the International Classification of Diseases 10th edition (ICD-10) [12]. For example, cerebral infarction (lacunar) was standardized as lacunar infarction. Next, the standardized disease names were classified for statistical analysis. For example, bile duct stones, bile duct dilatation, and cholangitis were categorized into bile duct disease.

There were problems with the standardization of the TCM syndrome diagnosis such as classification confusion and different names of the same syndrome. In these circumstances, the compound syndrome was divided into two or several single syndromes and standardized according to Clinic terminology of traditional Chinese medical diagnosis and treatment • Syndromes (GB/T16751.2- 1997) [13] and syndrome element syndrome differentiation (SESD) [14]. For example, the "qi and yin deficiency with blood stasis" syndrome was divided into qi deficiency, yin deficiency, and blood stasis.

\subsection{Data Analysis}

2.3.1. Descriptive Analysis. Microsoft Excel 2019 was used to count the gender, age, distribution of visits, and frequency of comorbidities of all patients in the included case data. Continuous variables with normal distribution were represented by mean \pm standard deviation (SD) and the continuous variables with nonnormal distribution were represented by median and interquartile ranges (M (Q1, Q3)). Categorical variables were described by frequency and percentage (n (\%)).

2.3.2. Construction of Neural Network Model. In this study, Python 3.6 was used to construct a fully connected neural prediction model from TCM syndromes to comorbidities. A fully connected neural network is the oldest data mining model, which has the most network parameters and the largest amount of computation. It consists of an input layer, hidden layer, and output layer, and the "neurons" in each layer are interconnected with the neurons in the next layer in a fully connected mapping model [15]. The input layer is the independent variable. The output layer is the dependent variable. The hidden layer and the number of nodes determine the complexity of the network model. The neural network first randomly assigns weights to the independent variables and then compares the predicted results with the known results. The prediction error of the hidden nodes in the upper layer is estimated by the prediction error of the output layer, and the error is back-propagated to the input 
layer from back to forward layer by layer, so as to adjust the link weight and find the best diagnostic model.

In this study, a four-layer fully connected neural network model was adopted. Descriptive analysis results were used to select representative syndromes and comorbidities. Label encoding of TCM syndrome diagnosis information was taken as the input layer and main diabetic comorbidities as the output layer, and the hidden layer was set as 2 layers (Figure 1).

In order to adapt to the neural network model, the relationship data of "multiple syndromes-multiple comorbidities" in the original data was split to form the data tables of "single syndrome" and "single comorbidity" in this study. The two tables were then fully joined to form the "syndromecomorbidity" data table (Figure 2). The single syndrome corresponds to the neural network's input layer, and the single comorbidity corresponds to the neural network's output layer.

When the neural network model was trained, more than $80 \%$ of samples were randomly selected from the "syndrome-comorbidity" data set as the training set, and the rest of the data selected as the test set. The training was considered complete when the calculated results of the model loss function became relatively stable. Finally, a neural network model for predicting the comorbidities of diabetes was established using TCM syndrome patterns. The calculation results of the final model in the prediction set were taken out to calculate the predictive probability of different syndrome groups for comorbidities.

\section{Results}

3.1. General Information. A total of 3413 medical records of 995 T2DM patients were included in this study, which comprised 158 types of diabetic comorbidities and 188 types of TCM syndromes. Of all patients, 470 were male (47.24\%) and 525 were female $(52.76 \%)$. In terms of age, the patients were mainly distributed in the range of 40 to 80 years old, including 465 (46.73\%) aged $40-59$ years and 374 (37.59\%) aged 60-79 years. The number of visits ranged from 1 to 36 , with a median of $2(1-4)$ visits, and the number of patients with 1-3 visits was the largest. Patients were mainly complicated with 1-2 diseases and were mostly associated with 12 syndromes (Table 1 ).

The top 10 comorbidities included hypertension, renal insufficiency, disorder of lipid metabolism, liver disease, neuropathy, heart disease, gastrointestinal disease, cerebral infarction, retinopathy, and biliary tract disease, accounting for $82.36 \%$ of all comorbidities (Figure 3). Compared with other age groups, there were more patients with liver diseases and gastrointestinal diseases in the 18-39 age group, and the number of patients with cardiovascular and cerebrovascular diseases increased significantly with age (Figure 3(a)). In terms of gender distribution, there were far more males than females in patients with liver disease (Figure 3(b)). The most common TCM syndromes included blood stasis, yin deficiency, qi deficiency, fire or heat, stomach heat, phlegm, spleen deficiency, liver constraint, dampness, and damp-heat (Figure 4). The syndrome manifestations of patients aged 18-39 years were mainly excess syndromes, such as blood stasis, fire or heat, stomach heat, phlegm, and liver constraint. The elderly over 80 years old showed obvious qi deficiency, yin deficiency, and blood stasis (Figure 4(a)). Female patients with qi deficiency, yin deficiency, and blood stasis were slightly higher in number than males with those symptoms (Figure 4(b)).

\subsection{Syndrome Characteristics of Different Comorbidities.} This study conducted a statistical analysis on the syndrome distribution characteristics of the top 10 comorbidities (Figure 5). It was found that there were obvious differences in the distribution characteristics, location, and nature of syndromes of different diseases. For example, in patients with diabetes mellitus complicated with hypertension, patterns of deficiency and excess were present in their syndrome manifestations. Deficiency syndromes were mainly yin deficiency and qi deficiency. The excess syndromes were fire or heat, blood stasis, phlegm, and yang hyperactivity. The syndromes of patients with renal insufficiency were mainly qi deficiency, blood stasis, yin deficiency, and fire (heat). At the same time, the syndrome characteristics of turbidity, toxin, dampness, and water retention were highlighted. Patients with liver disease mostly had liver and stomach organ lesions. The main syndrome characteristic was the disorder of $q i$ movement in the middle jiao, and the syndrome manifestations included stomach heat, liver constraint, fire or heat, phlegm, and qi stagnation. Patients with neuropathy mainly showed deficiency syndrome and stasis syndrome, including qi deficiency, blood stasis, yin deficiency, and coldness. The disease location of combined heart disease was mainly in the heart, and the main syndromes included $q i$ deficiency, yin deficiency, blood stasis, and yang deficiency.

\subsection{Evaluation of "Syndrome-Comorbidity" Neural Network} Model. There was a total of 4375 "syndrome-comorbidity" data cases for diabetes, with 188 syndrome diagnoses, which formed a $4375 \times 188$ label-encoding characteristic matrix. The top 10 comorbidities of diabetes were selected as the output label Y, forming a $4375 \times 10$ Label-encoding matrix. The original data was divided into a training set and a test set. 3875 cases of data were randomly selected from the feature matrix $\mathrm{X}$ as the training set and the remaining data as the test set. After repeated training of the model, convergence was reached at 1400 runs (Figure 6).

MSE is the mean square error, which is the average value of the square of the distance between the predicted value $f(x)$ of the model and the real value $y$ of the sample. It is a type of loss function which is used to evaluate the degree of inconsistency between the predicted value $f(x)$ of the model and the real value $y$.

\section{4. "Syndrome-Disease" Predictive Relationships for Major} Comorbidities. Through detailed analysis of the prediction results of the model, it was found that the accuracy of the model in predicting the seven comorbidities of renal insufficiency, gastrointestinal disease, hypertension, 


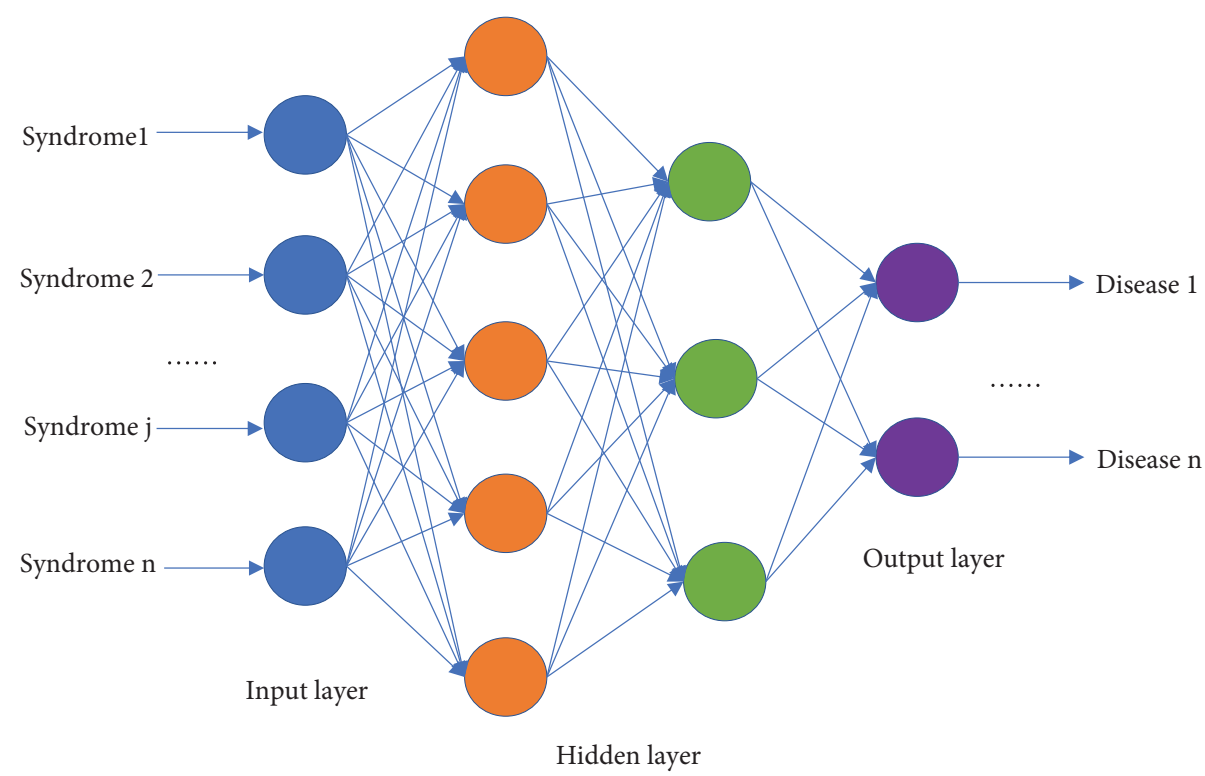

FIgURE 1: Neural network model of predicted TCM syndromes and comorbidities.

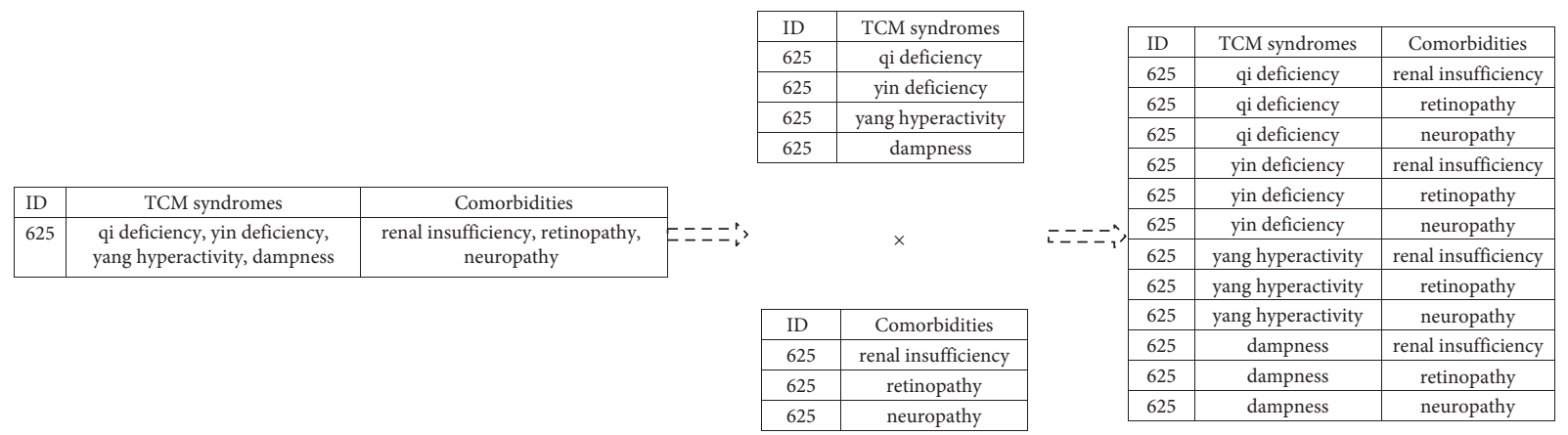

FIGURE 2: Example of "syndrome-comorbidity" construction process.

neuropathy, heart disease, liver disease, and lipid metabolism disorder could reach 70 90\%, while the prediction accuracy for cerebral infarction was about $50 \%$, and the prediction accuracy for retinopathy and biliary tract disease was below 50\% (Figure 7). The results showed that different characteristic syndrome groups could be used to predict the corresponding comorbidities.

Renal insufficiency was the most predictive comorbidity. Patients with TCM syndrome of blood amassment in the lower jiao were diagnosed with renal insufficiency with 95\% accuracy. Patients with syndrome groups of kidney qi deficiency, turbid, toxic, and obstruction in meridians and collaterals were diagnosed with renal insufficiency with a sensitivity of $87 \%$. It could also be seen from other TCM syndrome groups that the syndrome was dominated by interior patterns, and patients with turbidity, toxin, stasis, and deficiency of the kidney and spleen had a higher probability of being diagnosed with combined renal insufficiency.

The gastrointestinal disease was also comorbidity with characteristic syndrome groups and high accuracy of prediction. Patients with spleen deficiency combined with ascending counterflow of stomach qi and cold-damp patterns were diagnosed with gastrointestinal lesions with 92\% sensitivity. The syndrome group of ascending counterflow of stomach qi and deficiency-coldness of the spleen and stomach were diagnosed with diabetes combined with the gastrointestinal disease with $91 \%$ sensitivity. It could also be observed from the other TCM syndrome groups that the main locations of disease were in the stomach, spleen, and middle jiao, and patients with the syndrome groups of deficiency, qi counterflow, coldness, and blood stasis had a high probability of being diagnosed with diabetes complicated with gastrointestinal disease.

For patients with hypertension, the probability of the syndrome group predicting the comorbidity was also relatively high. 92\% of patients with TCM syndrome group of spleen heat and exuberance of heart fire were diagnosed with type 2 diabetes complicated with hypertension. 89\% of patients with the syndrome group of liver wind, ascendant hyperactivity of liver yang, liver yin deficiency, and kidney yin deficiency were diagnosed with diabetes combined with hypertension. $86 \%$ of patients with TCM syndrome group of liver yin deficiency, kidney yin deficiency, disharmony of the 
TABLE 1: General information of type 2 diabetes patients with comorbidity.

\begin{tabular}{lc}
\hline Variable & Value \\
\hline Gender, $n(\%)$ & $470(47.24)$ \\
$\quad$ Male & $525(52.76)$ \\
Female & $56(46,65)$ \\
Age (years), M (Q1, Q3) & \\
Age (years), $n(\%)$ & $130(13.06)$ \\
$18-39$ & $465(46.73)$ \\
$40-59$ & $374(37.59)$ \\
$60-79$ & $26(2.61)$ \\
$\geq 80$ & $2(1,4)$ \\
Frequency of visit, M (Q1, Q3) & \\
Frequency of visit, $n(\%)$ & $717(73.31)$ \\
$1-3$ & $144(14.72)$ \\
$4-6$ & $42(4.29)$ \\
$7-9$ & $75(7.67)$ \\
$\geq 10$ & \\
Comorbidity, $n(\%)$ & $3019(86.82)$ \\
$1-2$ & $396(11.39)$ \\
$3-4$ & $62(1.78)$ \\
$\geq 5$ & \\
TCM syndrome, $n(\%)$ & \\
$1-2$ & \\
$3-4$ & \\
$\geq 5$ & $1629(24.33)$ \\
\hline
\end{tabular}

M: median; Q1: lower quartile; Q3: upper quartile.

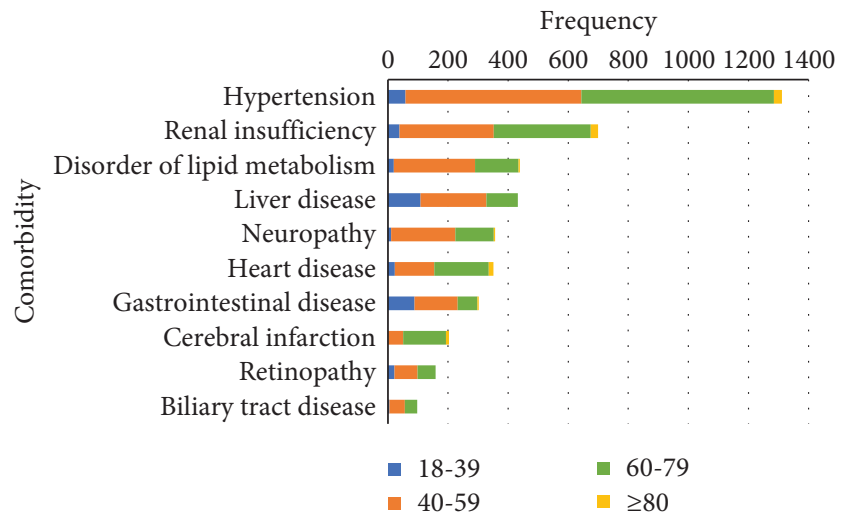

(a)

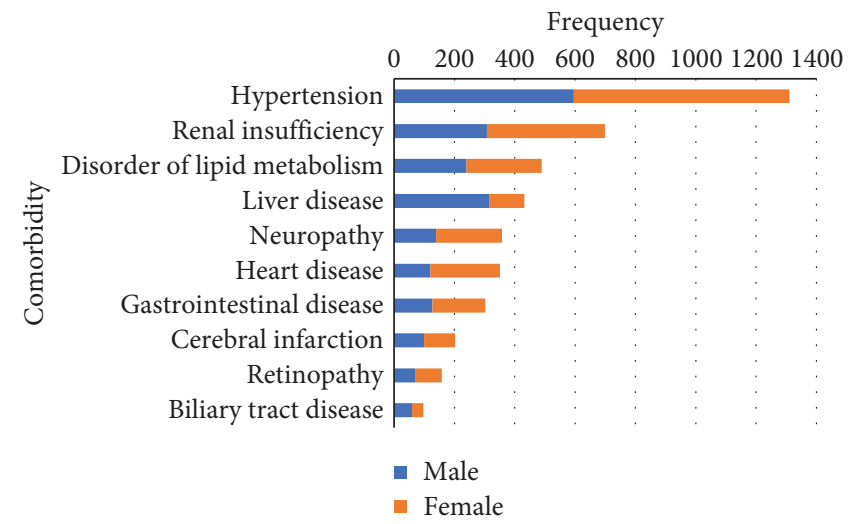

(b)

Figure 3: Distribution of comorbidities. (a) Age. (b) Gender.

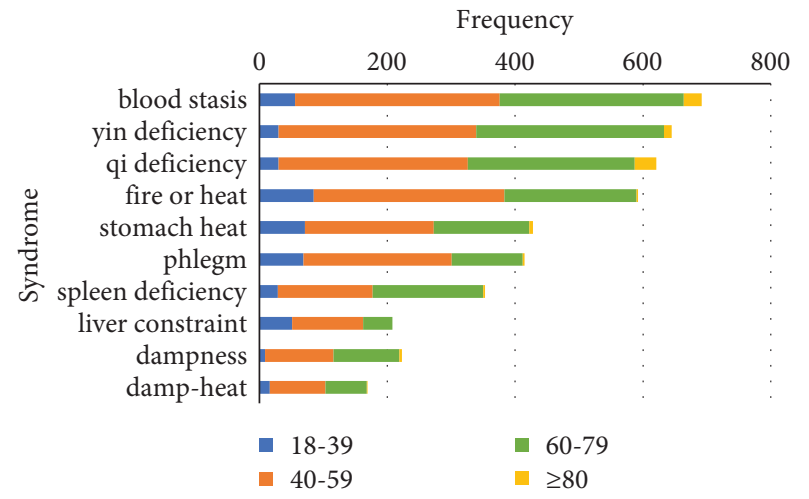

(a)

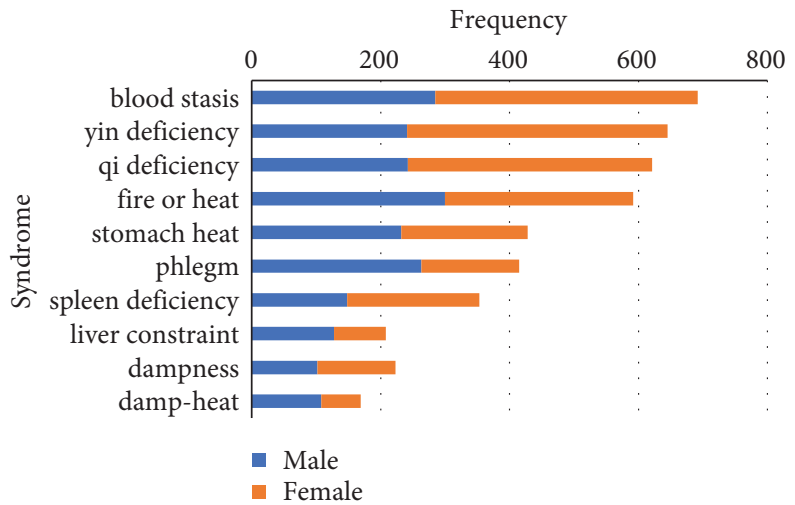

(b)

FIgURE 4: Distribution of syndromes. (a) Age. (b) Gender. 


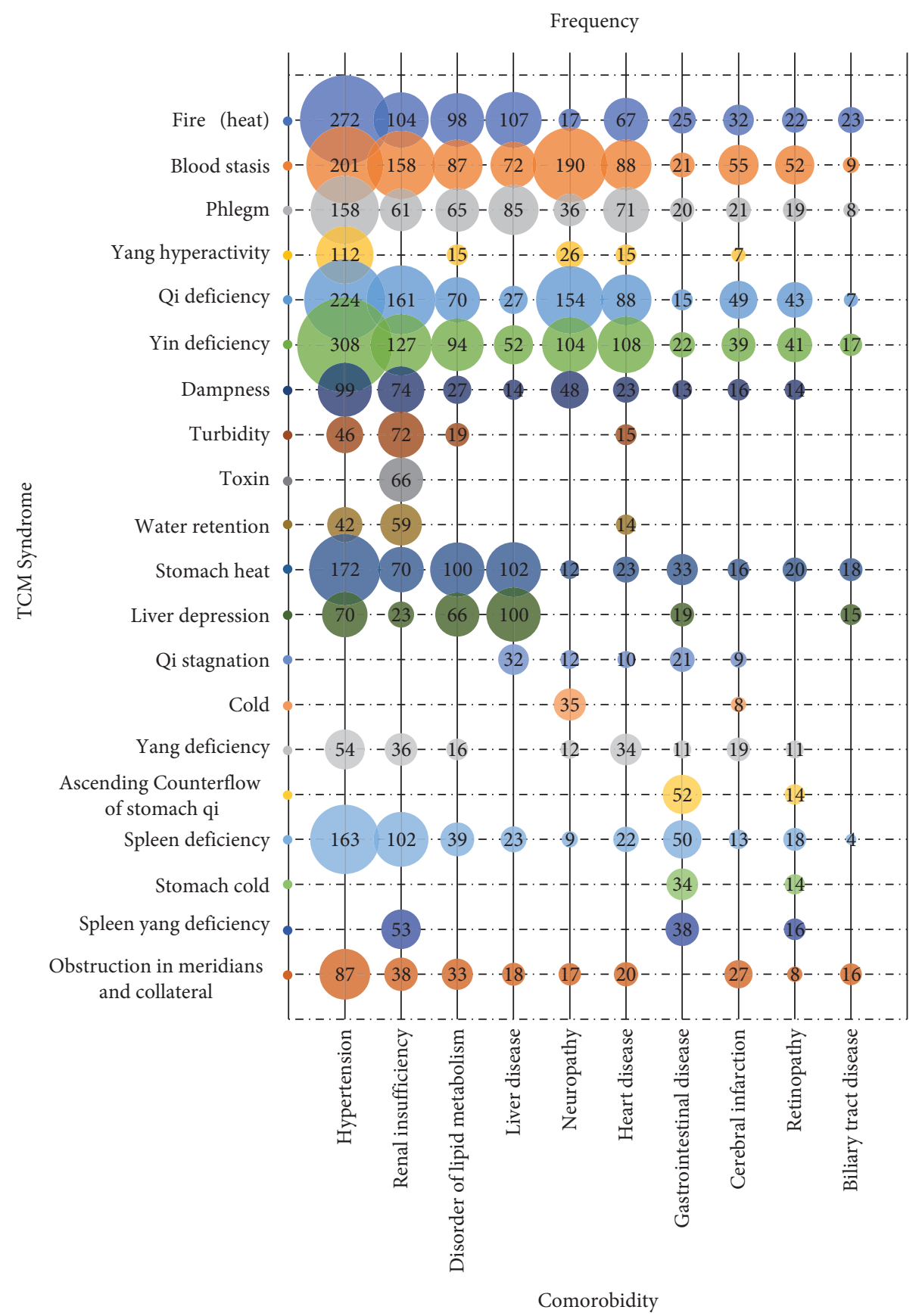

Figure 5: Syndrome distribution of comorbidities.

chong and ren mai, and yang floating were diagnosed with hypertension. It could also be seen from the other TCM syndrome groups that a portion of patients with liver or kidney as the main location of disease and deficiency or wind as the main nature of disease were diagnosed as diabetes complicated with hypertension.

The results of this study showed that different syndrome groups could predict corresponding comorbidities. The syndromes of fire or heat, qi stagnation, blood stasis, and dampheat were associated with the diagnosis of diabetes complicated with liver disease. A high portion of patients who had a location of disease mainly in the heart and kidney, nature of the disease being deficiency and coldness, were diagnosed with diabetes complicated with heart disease. Those with blood stasis, coldness, and deficiency as the main syndrome manifestations were diagnosed with type 2 diabetes complicated with neuropathy with high sensitivity. Damp retention in the middle jiao was the characteristic symptom in the diagnosis of type 2 diabetes mellitus combined with lipid metabolism disorder. However, among all TCM syndrome groups, the probability of being diagnosed with diabetes combined with cerebral infarction, retinopathy, and biliary tract disease was relatively low, and no syndrome group with a prediction rate of more than $60 \%$ was found. 59\% of those with TCM syndrome of kidney essence 


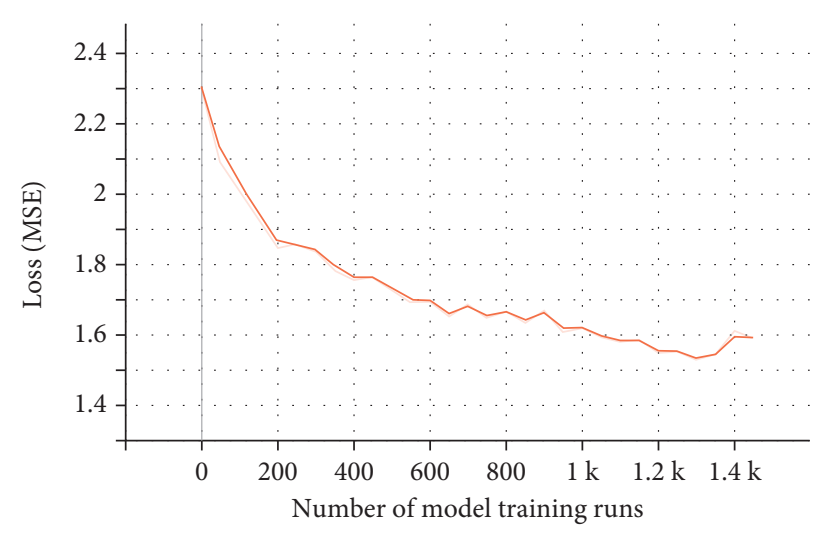

FIGURE 6: Evaluation of predictive neural network of TCM syndromes and comorbidities in diabetic patients.

deficiency and cerebrospinal damage were diagnosed with cerebral infarction, and $56 \%$ of patients with the independent syndrome of wind were diagnosed with diabetes mellitus combined with cerebral infarction.

\section{Discussion}

4.1. Distribution Characteristics and Overall Relationship between Diabetic Comorbidities and TCM Syndromes. The results of this study showed that the common syndromes of diabetic patients with comorbidity were a combination of deficiency and excess. Excess syndrome mainly consists of heat syndrome, blood stasis syndrome, and phlegm syndrome. Qi deficiency and yin deficiency were the main patterns of the deficiency syndrome. The number of younger diabetic patients with digestive system diseases was higher in comparison to the elderly, who were more likely to be complicated with cardiovascular and cerebrovascular diseases. In terms of syndrome manifestation, younger patients showed a higher incidence of the excess syndrome, while the elderly patients showed a higher incidence of deficiency syndrome and blood stasis. In terms of gender distribution, male patients were more likely to be complicated with liver diseases, while patients with qi deficiency, yin deficiency, and blood stasis syndrome were mostly female. According to TCM, diabetes is a chronic progressive disease, and the pathogenesis is a deficiency-excess complex. The core pathogenesis of diabetes is "deficiency, heat, and blood stasis" $[16,17]$. At the same time, phlegm is also an important pathological factor in diabetes mellitus [18]. This is consistent with the results of this study. In addition, constraintheat is the main pathogenesis in the early stage of diabetes and gradually progresses to the stage of deficiency and detriment with the development of the disease [19]. The influence of age on the distribution of diseases and syndromes is related to the modern living and working environment, physique, course of the disease, and other factors. Gender distribution is a dividing factor that may relate to physiology and living environment differences.

The results of this study showed that there were obvious differences in the syndrome distribution characteristics of different comorbidities, and the prediction result of the neural network showed that the prediction of comorbidities by characteristic syndrome group had high accuracy. Studies have shown that untreated type 2 diabetes is prone to various acute or chronic complications when the body is in a high glucose state for a prolonged period of time [20], and with the progression of the disease, the distribution characteristics and symptoms change accordingly [21]. This shows that there is a close correlation between different comorbidities and TCM syndromes. Different pathogenesis often leads to the occurrence of different comorbidities, the characteristics of the comorbidities are significant in the manifestations of TCM syndromes, and there will be some syndrome groups with very obvious characteristics in different diabetic comorbidities. Characteristic syndrome groups can be an important factor in distinguishing the corresponding comorbidities.

\subsection{Syndrome Characteristics of Different Comorbidities}

4.2.1. Hypertension. The results of this study showed that hypertension was the most common comorbidity. Its TCM syndromes were characterized by liver wind, fire or heat, yin deficiency, and disharmony of the chong and ren mai, and the location of the disease was mainly reflected in the kidney and liver. Patients with TCM syndrome group of spleen heat and exuberance of heart fire were diagnosed as type 2 diabetes complicated with hypertension with a sensitivity of $91 \%$. TCM considers "liver-kidney yin deficiency" as the root (the mean) and the "wind, fire, phlegm, and blood stasis" as the branch (the secondary) in terms of hypertension pathogenesis [22-24]. Dating back to as early as in The Yellow Emperor's Inner Classic: Basic Questions, there was a description of the pathogenesis of hypertensive vertigo: "All wind with vertigo is ascribed to the liver". Meanwhile, disharmony of the chong and ren mai is also a common symptom of hypertension in perimenopausal women $[25,26]$. With the increase of blood glucose, diabetes patients usually suffer from hunger, weight loss, constipation, frequent urination, and other symptoms, which is the main manifestation of "Stagnancy of Er Yang", just like what is described in The Yellow Emperor's Inner Classic: "Disorder of Stomach may be Affected by Disorders of the Heart or Spleen" [27]. Exuberant heat in the heart and spleen combined with emotional depression leads to the binding constraint of liver 


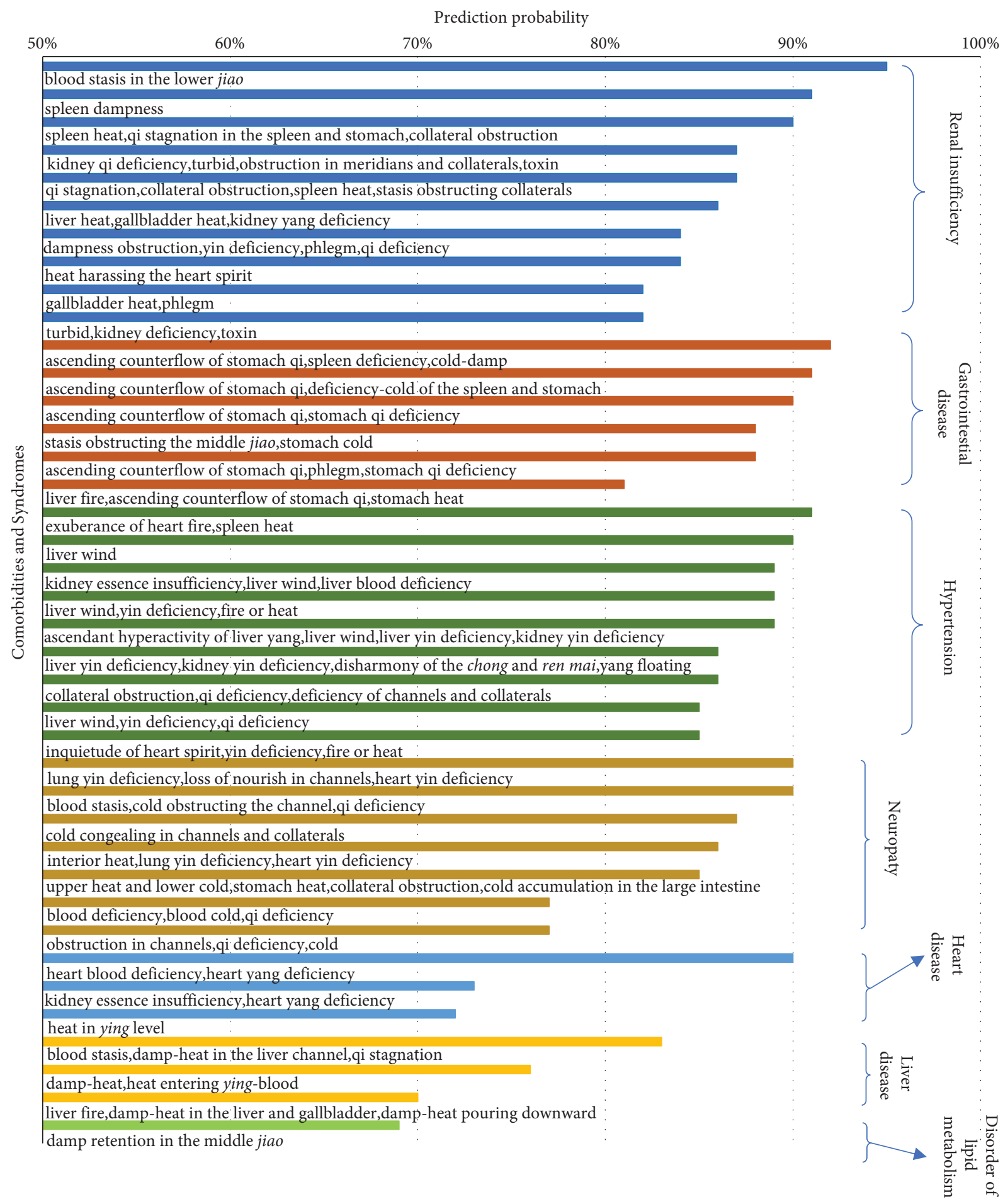

FIGURE 7: Probability of predicting comorbidity by different syndrome groups.

$q i$ and induces ascendant hyperactivity of liver yang and results in the occurrence of hypertension. The prediction results of hypertension in this study are consistent with Traditional Chinese medicine theory and clinical practice.

4.2.2. Renal Insufficiency. Renal insufficiency is also common comorbidity of diabetes. The results of this study showed that in addition to the syndrome of "blood stasis, heat, and deficiency", the syndromes of turbidity, poison, and dampness were also associated with renal insufficiency. At the same time, patients with TCM syndrome of blood amassment in the lower jiao were diagnosed with renal insufficiency with $95 \%$ sensitivity. Those with the syndrome group of kidney qi deficiency, turbid, toxic, and obstruction in meridians and collaterals were diagnosed with diabetes complicated with renal insufficiency with a sensitivity of 
$87 \%$. The kidney is located in the lower jiao. With further development of the disease, kidney essence is damaged, causing the failure in $q i$ transformation, which creates difficulty in distinguishing the clear and metabolic turbidity, leading to the accumulation of turbid pathogens and water-dampness and formation of turbid-toxin. Turbidtoxin obstruction in the collateral and consequent unsmooth blood circulation results in water retention which then leads to edema and eventually results in kidney disease [28]. Meanwhile, turbidity-toxin is closely related to glucose toxicity and lipid toxicity in diabetes mellitus [29]. Removing turbidity and resolving toxins are also important priorities in treating diabetic nephropathy [30-32]. The occurrence of related diseases can be actively prevented according to the symptoms, which is also of great significance in controlling the progression of diabetes.

4.2.3. Neuropathy. The characteristic syndromes of diabetes complicated with neuropathy were malnutrition of channels and coldness coagulation in the meridians and collaterals. The syndrome group of "blood stasis, qi deficiency, and cold coagulation in meridians and collaterals" could predict diabetic neuropathy with an accuracy of $90 \%$. In the early stage of diabetes, "heat syndrome" is the main syndrome, whereby the pathogenic heat damages the essential qi of zang-fu organs, especially the spleen. Spleen yang deficiency cannot reach the end of the limbs, resulting in a counterflow coldness in the four limbs. The coldness congealment leads to blood stasis, which results in blood bi and coldness coagulation in meridians and collaterals [33]. This is also consistent with the understanding of diabetes complicated with neuropathy in TCM. It is believed that the location of this disease is mainly in the channels and collaterals, involving the liver, kidney, and spleen. Qi and blood deficiency are considered as the root of pathogenesis and the blood stasis obstructing collaterals as the branch [34].

4.2.4. Other Comorbidities. In addition, there was also a close relationship between TCM syndromes and other comorbidities. Different comorbidities have their own syndrome groups with obvious characteristics. For example, the syndrome characteristics of liver diseases are damp-heat, qi stagnation, and blood stasis. The binding constraint of liver qi and liver-gallbladder damp-heat are two new syndromes that have evolved in modern life, while stasis syndrome is often the key to disability and lifethreatening diabetic complications [35]. The syndrome characteristics of combined heart disease are mainly heart qi deficiency or heart yang deficiency. The characteristic syndromes of patients complicated with gastrointestinal disease were ascending counterflow of stomach qi, stomach qi deficiency, and stomach coldness. The syndrome of disorder of lipid metabolism is characterized by dampness obstruction in the middle jiao. It can be seen that with the progression of diabetes, the TCM syndromes also evolve correspondingly, and the TCM syndromes show different manifestations during the occurrence of different comorbidities, indicating that the comorbidities are closely related to the TCM syndromes. It is of great practical significance to be able to predict the associated comorbidities through characteristic syndromes in the diagnosis, treatment, and prevention of diabetes mellitus.

\section{Conclusion}

In summary, this paper introduced a fully connected neural network approach to the study of the association between type 2 diabetic comorbidities and TCM symptoms with the construction of a "TCM symptom-comorbidity" prediction model. Different kinds of comorbidities were characterized by "deficiency, heat, phlegm, and blood stasis" in the viscera of diabetic patients, such as the heart, spleen, stomach, and kidney. According to the characteristics of the disease, different syndrome elements were combined to form characteristic syndrome groups. The corresponding comorbidities of diabetes were predicted based on the characteristics of the syndrome groups with a high degree of accuracy. The construction of the "TCM syndromecomorbidity" prediction model may be very helpful in assisting the early diagnosis of diabetic comorbidity. However, due to the limitations of the data, only ten common comorbidities were included in this study. The data volume will be expanded in the future to further determine the relationship between type 2 diabetic comorbidities and different TCM syndromes.

\section{Data Availability}

The data used to support the findings of this study are included within the article.

\section{Conflicts of Interest}

The authors declare that there are no conflicts of interest.

\section{Acknowledgments}

This study was supported by the National Natural Science Foundation of China (No. 81774158), China Academy of Chinese Medical Sciences Innovation Fund (No. CI2021A00509), and "Three Famous Project": Method Innovation of Clinical Appraisal of Chinese Medicine, Acupuncture, and Moxibustion by Team of Liu Baoyan, Principal Researcher of China Academy of Chinese Medical Sciences (SZSM201612001).

\section{References}

[1] T. Zhang, Q. He, Y. Liu, Z. Chen, and H. Hu, "Efficacy and safety of resveratrol supplements on blood lipid and blood glucose control in patients with type 2 diabetes: a systematic review and meta- analysis of randomized controlled trials," Evidence-Based Complementary and Alternative Medicine, vol. 2021, Article ID 5644171, 15 pages, 2021.

[2] International Diabetes Federation, IDF Diabetes Atlas 9th, International Diabetes Federation, Brussels, Belgium, 2019, https://www.diabetesatlas.org. 
[3] J. N. Struijs, C. A. Baan, F. G. Schellevis, G. P. Westert, and G. A. van den Bos, "Comorbidity in patients with diabetes mellitus: impact on medical health care utilization," $B M C$ Health Services Research, vol. 6, no. 1, p. 84, 2006.

[4] A. Manoharan and M. Santhanakumar, "Anti-diabetic evaluation of hydro alcoholic extract of herbal plant oorithal thaamarai chooranam (Otc) on streptozotocin (STZ) induced Type 2 diabetes mellitus in wistar albino rats," Journal of Traditional and Integrative Medicine, vol. 4, no. 2, pp. 519525, 2021.

[5] N. Nayebi, A. Esteghamati, A. Meysamie et al., "The effects of a Melissa officinalis L. based product on metabolic parameters in patients with type 2 diabetes mellitus: a randomized double- blinded controlled clinical trial," Journal of Complementary \& Integrative Medicine, vol. 16, no. 3, 2019.

[6] M. Seyed Hashemi, N. Namiranian, H. Tavahen et al., "Efficacy of pomegranate seed powder on glucose and lipid metabolism in patients with type 2 diabetes: a prospective randomized double- blind placebo-controlled clinical trial," Complementary Medicine Research, vol. 28, no. 3, pp. 226233, 2021.

[7] K. Eqbal, M. A. Alam, M. A. Quamri, G. Sofi, and M. D. Ahmad Bhat, "Efficacy of Qurs-e-Gulnar in ziabetus (type 2 diabetes mellitus): a single blind randomized controlled trial," Journal of Complementary and Integrative Medicine, vol. 18, no. 1, pp. 147-153, 2021.

[8] M. A. Morowatisharifabad, M. Asadpour, M. A. Zakeri, and M. Abdolkarimi, "The effect of integrated intervention based on protection motivation theory and implementation intention to promote physical activity and physiological indicators of patients with type 2 diabetes," BioMed Research International, vol. 2021, Article ID 6637656, 8 pages, 2021.

[9] National Center for Complementary and Alternative Medicine National Institutes of Health, What is Complementary and Alternative Medicine?, https://nccam.nih.gov/health/ whatiscam, 2021.

[10] T. T. Deng, Criteria for TCM Syndromes, Guangdong Science and Technology Press, Guangzhou, China, 1990.

[11] R. H. Zhang and B. Li, "The connotation and academic viewpoints of the essence of syndrome," Chinese Journal of Integrated Traditional and Western Medicine, vol. 29, no. 04, pp. 375-378, 2009.

[12] World Health Organization, International Statistical Classification of Disease and Related Health Problems, Tenth Revision (ICD-10). World Health Organization, Geneva, Switzerland, 1992.

[13] The State Bureau of Quality and Technical Supervision, Clinic Terminology of Traditional Chinese Medical Diagnosis and Treatment, Standards Press of China, Beijing, China, GB/T 16751.2-1997, 1997.

[14] W. F. Zhu, Syndrome Element Syndrome Differentiation (SESD), People's Health Publishing House, Beijing, China, 2008.

[15] K. Y. Hsu, H. Y. Li, and D. Psaltis, "Holographic implementation of a fully connected neural network," Proceedings of the IEEE, vol. 78, no. 10, pp. 1637-1645, 1990.

[16] J. Zhao, X. Feng, X. Tong et al., "TCM core pathogenesis and basic therapeutic methods for diabetes," Beijing Journal of Traditional Chinese Medicine, vol. 38, no. 01, pp. 3-6, 2019.

[17] Y. Yang, Z. X. Hu, and J. H. Liu, "Research on the Analysis of the pathogenesis of type 2 diabetes based on evaluating syndrome by drug intervention," Clinical Journal of Traditional Chinese Medicine, vol. 33, no. 01, pp. 11-16, 2021.
[18] P. Zhang, F. Sun, S. Wang et al., "Pang guoming's experience in treating type 2 diabetes from phlegm," Journal of Traditional Chinese Medicine, vol. 60, no. 18, pp. 1546-1549, 2019.

[19] Diabetes Branch of Chinese Medical Association, "Chinese guidelines for prevention and treatment of type 2 diabetes (2020 edition)," Chinese Journal of Endocrinology and Metabolism,vol. 37, no. 4, pp. 311-398, 2021.

[20] W. Liang, L. Kang, X. Meng et al., "Research progress on the relationship between intestinal microflora imbalance and complications of type 2 diabetes mellitus," Shandong Medical Journal, vol. 57, no. 29, pp. 107-109, 2017.

[21] Y. Xing, M. Pi, R. S. Zhang, and T. Wen, "Study on the TCM syndromes evolution and Chinese herbal characteristics of type 2 diabetes patients with different courses of disease in TCM heat stage: a real-world study," Evid Based Complement Alternative Medicine, vol. 2021, Article ID 1282957, 12 pages, 2021.

[22] Y. Deng, L. Chang, F. Qi, S. Li, and Y. Cui, "Thinking about curing the hypertension illness through wind-phlegm and blood stasis blocking the collaterals," Chinese Archives of Traditional Chinese Medicine, vol. 29, no. 04, pp. 750-751, 2011.

[23] L. F. Cui, J. H. Luo, and Z. Y. Li, “Qiu baoguo's experience in treating refractory hypertension," Acta Chinese Medicine, vol. 29, no. 10, pp. 1447-1448, 2014.

[24] B. Du, G. Zhao, J. Cheng, and J. Qu, "Effect and mechanism of liu-wei-di-huang pill on vertigo in hypertensive patients with yin deficiency of liver and kidney," Journal of Chinese Medicinal Materials, vol. 06, pp. 1509-1513, 2021.

[25] L. Su, H. Jin, L. Zheng, Z. Liu, and S. Liu, "On the disharmony of thoroughfare vessel and conception vessel is the key to the pathogenesis of perimenopausal hypertension," Traditional Chinese Medicinal Research, vol. 30, no. 01, pp. 1-3, 2017.

[26] Y. M. Wang, F. J. Liang, and X. J. Feng, "Efficacy of TCM syndrome differentiation in treatment of primary hypertension," Chinese Journal of Integrative Medicine on CardioCerebrovascular Disease, vol. 14, no. 17, pp. 2028-2030, 2016.

[27] Q. Fu, S. Wang, Y. Xiao et al., "Exploration of professor lv renhe's academic thoughts on treating diabetes mellitus by staging treatment," World Chinese Medicine, vol. 12, no. 01, pp. 21-24, 2017.

[28] X. W. Zhang, H. F. Liu, and X. H. Zhang, "Analysis of pathogenesis level and treatment differentiation of diabetic nephropathy," Journal of Traditional Chinese Medicine, vol. 58, no. 05, pp. 390-393, 2017.

[29] S. T. Wu, "On the relevance of turbidity-toxin to glucotoxicity and lipotoxicity in diabetes mellitus," Journal of Traditional Chinese Medicine, vol. 09, pp. 647-649, 2004.

[30] L. Peng, Q. Tang, J. Liang, and Q. Xu, "Experience in the treatment of early type 2 diabetes mellitus from a discussion of turbid toxins trapped in the spleen," Global Traditional Chinese Medicine, vol. 13, no. 12, pp. 2111-2113, 2020.

[31] X. Wei, L. Yan, J. Lv, W. Xu, and D. Li, "Example of professor li diangui in treating chronic kidney disease by using zhuodu theory," Chinese Medicine Modern Distance Education of China, vol. 13, no. 15, pp. 40-43, 2015.

[32] S. Chen and S. T. Wu, "Overview of the treatment of diabetic nephropathy from the perspective of turbid toxicity," Hunan Journal of Traditional Chinese Medicine, vol. 35, no. 04, pp. 168-169, 2019.

[33] X. Zhao, Q. Yu, Y. Liu et al., “Tong xiaolin's experience in the management of diabetic peripheral neuropathy," Journal of Traditional Chinese Medicine, vol. 54, no. 10, pp. 882-883, 2013. 
[34] G. M. Pang, Y. Yan, P. Zhu et al., "Clinic draft specification of traditional Chinese medicine about diabetic peripheral neuropathy," China Journal of Traditional Chinese Medicine and Pharmacy, vol. 35, no. 02, pp. 260-264, 2010.

[35] Z. Q. Liu, "Experience of TCM syndrome differentiation and treatment of type 2 diabetes," Journal of Sichuan of Traditional Chinese Medicine, no. 11, pp. 20-22, 2006. 